\title{
COMMUNIQUER DANS DES ORGANISATIONS SEMI-PUBLIQUES : TECHNICIEN OU INTERMÉDIAIRE SOCIAL?
}

\begin{abstract}
Jos Pirson ${ }^{1}$
Durant la dernière décennie, un certain nombre d'organisations publiques se sont restructurées; il en a été de même d'associations ou d'entreprises dans lesquelles « secteur privé » « secteur public » ont été amenés à s'investir. Dans ce contexte, le présent article prétend interroger quelques caractéristiques des fonctions et métiers de la communication : les évolutions analysées pourraient en effet être porteuses de renseignements pour l'analyse d'autres contextes ( public «pur », privé marchand ou non marchand...). L'hypothèse principale développée dans les pages qui suivent, consiste dans la reconnaissance d'un paradoxe : le modèle économique dominant exerce une emprise croissante sur la définition des tâches (la prégnance de la logique privée dans le secteur public); l'estompement des modèles culturels traditionnels normatifs favorise par contre la redéfinition de certaines fonctions de communication par les acteurs responsables de ces tâches (maîtrise de nouvelles zones d'incertitude).
\end{abstract}

1 Jos Pirson est directeur de l'Institut Libre de Formation Permanente (Namur), membre du LASCO et chargé de cours invité au Département de communication de l'Université catholique de Louvain

Recherches en communication, $\mathrm{n}^{\circ} 25$ (2006). 
Dans sa phase heuristique cet article part des indications données au fil d'entretiens semi-directifs par des professionnels de la communication sur leurs pratiques et sur la nature des processus de légitimation à l'œuvre. Nous sommes invités à considérer ces métiers selon un double axe diachronique (évolution de la «fonction communication » en organisation) ${ }^{1}$. et synchronique (combinaison de fonctions et de métiers pour constituer une profession $)^{2}$.

L'histoire comme méthode de reconstruction des faits sociaux est à considérer dans ses ambiguïtés et ses différentes facettes : lorsque il est fait état d'occultation versus dévoilement de la réalité organisationnelle à travers le métier de communicateur, nous pouvons estimer que la dynamique de recherche sur les métiers de la communication met elle-même en évidence certains traits et en néglige d'autres ${ }^{3}$.

La reconstruction opérée lors d'une rencontre entre professionnel(le)s de la communication et chercheurs a fait apparaître la triple influence de la culture sociétale (les « métiers reconnus » dans la société occidentale post-industrielle), de l'environnement direct ( l'effet de notoriété et la fiabilité de telle catégorie professionnelle par rapport à une autre) et du jeu structuré de l'action organisée ( les processus de défiance et de confiance, la reconnaissance ou la méconnaissance réciproque, les accords et désaccords entre partenaires)

1 J.L.MICHEL, Les professions de la communication : fonctions et métiers, Paris, Ellipses, collection Infocom, 1999. C.DUTERME, La communication interne en entreprise : l'approche de Palo Alto et l'analyse des organisations, Bruxelles, De Boeck Université, 2002

2 Comme nous le précisons un peu plus loin, les métiers correspondent à des segments d'activités sur base de profils précis de compétences. Les professions regroupent différents métiers et correspondent à des profils plus larges. On trouve des traces de cette logique normative dans la volonté d'identifier au sein de l'Union Européenne et, de manière plus globale, du Conseil de l'Europe différents niveaux de qualification et de compétences (12 niveaux dans un cadre appelé EQF, European Qualifications Framework)

3 Pour approfondir cette question le lecteur pourra se référer à différents ouvrages, par exemple P.DOCKES, B.ROSIER, “L'histoire ambiguë, Croissance et développement en question »,Paris, PUF, 1988. F.DOSSE, «L'empire du sens. L'humanisation des sciences humaines », Paris, La découverte, 1997. Voir également J.PIROTTE, "L'histoire entre mythe et vérité, Lieux de mémoire en Wallonie », Louvain la Neuve, Fondation Wallonne, 1999 ou encore J.PIRSON, «Construire du sens ? Perspectives épistémologiques... », Strasbourg, Revue des Sciences Religieuses, $\mathrm{n}^{\circ}$ 2, avril 2002,pp.204-217 
La légitimation comme processus exclut de considérer la légitimité comme une situation figée dans le temps ou comme phénomène d'attribution de sens indépendant d'un contexte sociétal précis. Différentes recherches menées sur l'induction du changement en organisation montrent l'intérêt d'une analyse des divers modes de reconnaissance, des phénomènes de cooptation, de coalition, des conflits, des crises de l'action organisée des conflits et des crises de l'action organisée ${ }^{1}$.

Les processus de légitimation renvoient par ailleurs aux divers phénomènes de changement comme apprentissages collectifs ( le changement culturel global est un apprentissage collectif) et à la justification des fonctions de communicateur dans un contexte sociétal précis. La définition de la pertinence nous renvoie aux recherches menées en analyse du langage chez des auteurs comme Peirce et Hempel : les phénomènes d'attribution de sens renvoient à la reconnaissance de savoirs partagés au sein de communautés qui savent sur quoi portent leurs accords et leurs désaccords ( en d'autres termes la validité reconnue des discours au sein d'un même monde culturel) $)^{2}$.

La question de la pertinence nous renvoie également à l'acceptation des postures sociales adoptées par ceux et celles qui prétendent communiquer en organisation : quelle est la capacité reconnue à ces acteurs de contribuer à l'action organisée, c'est à dire aux modes de coopération conflictuelle dans des jeux structurés de relations ? En d'autres termes, les acteurs sont-ils (elles) capables de contribuer à la régulation collective des séquences de relations sociales construites dans un environnement précis (qualifié d'interne, de semi-public, de public...)?

Il est utile d'introduire également une autre distinction importante pour le repérage empirique et l'analyse de la fonction de «communicateur » en organisation semi-publique: celle opérée dans différentes sphères d'activité entre des logiques de métiers et des logiques de professions $^{3}$. La logique de métiers correspond à la segmentation de

1 Ces observations ont pu être relevées par l'auteur dans l'étude de la relation entre transformation des systèmes d'éducation et de formation (des adultes en particulier)et transformation des fonctions de communication.

2 Voir le concept de pertinence chez PEIRCE et HEMPEL, Chez ce dernier, en particulier (1966), la pertinence renvoie à l'énoncé de propositions qui appartiennent à l'univers sémantique sous-jacent à la problématique décrite. Pour plus de précisions, v. J. PIRSON, op. iam cit.

3 En Communauté Wallonie-Bruxelles (Belgique francophone), nous assistons à un redéploiement de la Commission Consultative des Profils de Qualification (CCPQ) : 
fonctions particulières et très spécialisées ; elle coïncide avec la volonté des organisations représentatives des travailleurs ou du patronat d'identifier différents niveaux de compétences et de ressources dans la plupart des domaines d'activités connotées comme «manuelles » ou «intellectuelles».

La logique professionnelle correspond davantage à un regroupement de compétences différenciées : ce jeu à partir d'un ensemble de compétences permet à un acteur de développer sa surface sociale ( sa « zone d'incertitude » pour reprendre l'expression de l'analyse stratégique telle que l'ont élaborée Crozier et Friedberg). Cela signifie que dans le jeu structuré de l'action organisée, certains disposent de moyens d'action reconnus à la fois par les pairs et par le sommet directionnel' ${ }^{1}$.

En étudiant la légitimité des métiers de la communication, nous sommes dès lors amenés à réfléchir à un statut plus large, capable d'englober différentes compétences et donc différents métiers : c'est la signification principale de la reconnaissance professionnelle dans la société globale, notamment celle du chargé de la communication ou du directeur de la communication («DIRCOM»). La logique professionnelle n'est pas purement normative : elle s'inscrit dans l'évolution des métiers et la combinaison de ceux-ci dans l'évolution de professions en termes d'émergence reconnue ou de dévalorisation progressive. Nous allons tenter de comprendre le sens possible de cette évolution dans ce jeu croissant de l'emprise du modèle privé dans le domaine public et dans des entreprises qui se présentent comme « mixtes ».

\section{Chronique de changements non annoncés}

Aucun élément n'indique a posteriori une différence fondamentale entre les métiers étudiés à partir des contacts opérés : à tout le moins rien n'indique des définitions de postes variant en fonction du type d'organisation; les représentations des métiers semblent différer à partir des contextes et de l'évolution sociétale (contexte global économique et social). A partir de ce premier constat renforcé par plusieurs indices

celle-ci regroupe délégués du patronat et des syndicats dans la construction des différents profils de métiers, à l'instar de ce qui a été opéré en France (dans l'élaboration du Répertoire des Métiers ou ROME).

1 A ce propos, on consultera avec profit M.ANTOINE, D. DEFLANDRE, F.NAEDENOEN, N.RENIER, (sous la direction de F.PICHAULT), Faut-il brûler la gestion des compétences, Bruxelles, De Boeck, 2006 
concordants, nous pouvons parler d'un processus de différenciation de la fonction «communication » en organisation ${ }^{1}$.Nous partons d'une question essentielle : celle de la fonction par rapport au sommet de l'organisation et par rapport à un ensemble plus vaste d'acteurs qui forment système.

\section{La place centrale de la direction générale}

Les responsables de communication parlent de missions confiées par le sommet de l'organisation. Parmi ces missions, nous avons pu relever la tâche de «briefer correctement une agence de presse » ou « résoudre le problème de la façon de présenter les choses ». Ces indications n'invitent pas pour autant à adopter une vision manichéenne de la «vraie communication» face à la «fausse communication». Les femmes et hommes engagés comme professionnels en organisation font longuement état de questions éthiques et déontologiques : il s'agit de préoccupations qui ne relèvent pas de la pure rationalité technicienne ( «faire passer le message », « huiler les rouages de l'institution », à l'instar de cette traductrice-interprète qui avouait être « une boîte à traduire sans avoir d'état d'âme...peu importe le message à traduire »). Une de nos interlocutrices l'énonce clairement :

On m'a demandé de modifier les résultats d'une enquête stress... on correspond à ce moment à une vision stratégique, celle de la direction...On est un des éléments de la stratégie générale... C'est une des raisons pour lesquelles j'ai cherché à quitter ce poste et à changer...

Les personnes interrogées envisagent leur mission comme une action complexe liée à des compétences différenciées : les récits font état de précision dans certains éléments techniques lorsque le métier est lié à une activité assez définie (le journal d'entreprise, la politique de communication interne). La fonction parait liée à la politique globale

1 Nous avons construit la perspective de métiers émergents ou déclinants Cette proposition a été validée à partir d'un travail en sous-groupe avec quatre responsables de communication d'entreprise ; cinq autres contacts ont été opérés par la suite avec d'autres responsables de communication. De manière parallèle mais convergente au plan méthodologique dix autres entretiens ont pu être menés avec des étudiants de deuxième cycle, dans l'univers d'organisations à la frontière du secteur public et du secteur privé pour comprendre l'évolution des fonctions de communication. 
définie au sommet de l'organisation et déclinable selon différents degrés d'autonomie et elle exprime un lien fort vis-à-vis de la direction des ressources humaines ou de la direction générale ${ }^{1}$.

La dépendance des « stratégies d'organisation » ou des « décisions politiques au sommet »a été analysée dans des recherches menées sur l'usage des métaphores dans la désignation des fonctions de communication en entreprise ${ }^{2}$. L'élaboration de «campagnes», de «stratégies globales » incite trop souvent à parler d'efficacité technique plutôt que de capacité d'organiser la coopération entre les différents acteurs ${ }^{3}$. Dans deux entreprises, le personnel est envisagé comme un public à convaincre par des «actions ciblées » du bien fondé des décisions du sommet directionnel. Cette évolution a été constatée également dans des entreprises publiques de télécommunication lors du développement de campagnes pour l'usage du téléphone mobile : les acteurs sont considérés par des responsables des ressources humaines comme «un public interne » à convaincre et non comme partenaires à part entière de négociations au sein de l'organisation. Dans ce contexte particulier, la fonction de communication est connotée de manière instrumentale.

Certains responsables de communication montrent toutefois l'évolution des métiers et la prise au sérieux de compétences nouvelles d'organisation :

Au départ, on était fortement dépendants de la communication industrielle, dans une communication relativement instrumentalisée... puis on nous a demandé de huiler les rouages, de jouer le rôle de ciment du comité de direction. Aujourd'hui, nous arrivons plus au centre des décisions quand il faut arriver avec des choses bien préparées en négociation... Mais il y a toujours

1 Vont dans ce sens les affirmations recueillies durant l'été 2006 par les journalistes de Références, supplément hebdomadaire des publications du groupe de presse Roularta (L'Echo, Trends-Tendances et le Vif-l'Express) Cette convergence est intéressante bien que l'on ne puisse lui accorder le même statut au plan de la recherche

2 D'autres analyses ont permis d'identifier à ce propos les vocables guerriers utilisés dans les métiers de communication. Voir les numéros I et II de la revue Recherches en Communication, 1994, en particulier l'étude de Axel GRYSPEERDT, L'entreprise comme ambassade ou la métaphore de l'ambassadeur dans la communication institutionnelle op.cit., I pp.93-112

3 Voir en particulier A.GRYSPEERDT, Les fondements professionnels des relations publiques et de la communication d'entreprise, in Guide des médias, Zaventem, Kluwer Editorial, 1992 
le risque d'assumer une fonction de poubelle avec la mission d'assumer des problèmes non traités par ailleurs...C'est le lieu de la débrouille, mais cela signifie qu'il y a du changement dans le métier. Avant il fallait défendre l'image à tout prix, maintenant il y a plus un appui des choix stratégiques et de la manière d'aborder une négociation. Comment ? Là on est dans le flou... ! ${ }^{1}$.

Cet extrait aide à comprendre l'évolution et les transformations des métiers de la communication en organisation.. D'autres récits d'expériences vécues dans des organisations en pleine transformation permettent d'approcher l'évolution des fonctions de communication d'organisation : les fonctions de communicateur en interne comme en externe n'apparaissent plus simplement liées à « la défense de l'image de l'organisation quoi qu'il en coûte». Il s'agit davantage, pour les professionnels rencontrés, d'aider à comprendre les enjeux de changements ou de politiques menées au sommet stratégique de l'entreprise et de rendre compte de manière plus factuelle de la complexité de l'action organisée dans un contexte précis ${ }^{2}$.

Lorsqu'il a été demandé de préciser les contraintes, différents éléments sont apparus : la contrainte de temps (réagir dans l'immédiateté), l'envergure des contacts à prendre et le volume des informations à traiter par rapport à différents interlocuteurs. Ces contraintes sont gérables de manière différenciée en fonction du contexte (en dehors d'une situation de crise, la personne engagée dans la fonction dispose d'une certaine marge de manœuvre).

En résumé, les différents entretiens indiquent des positions variables par rapport au sommet stratégique : marge de manœuvre, capacité de négociation avec différentes instances ou imposition d'un mode

1 Entretien lors de la rencontre collective présentée ci-avant. L'acteur interrogé est responsable d'une équipe de communication interne et externe au sein d'une grande entreprise

2 Cette affirmation est étayée par le consensus observé au niveau des différents interlocuteurs. Par ailleurs les cinq entretiens publiés par le supplément références du magazine hebdomadaire Le Vif-l'Express du 29 juin au 28 juillet 2006 peuvent être interprétés dans le même sens : il s'agit de responsables de la communication dans cinq grandes entreprises publiques ou privées en Belgique. Les responsables interrogés insistent sur la précision des informations à recueillir et à transmettre, mais également sur la relation de confiance qui peut, en dernière instance, fonder la capacité de construire des actions de communication acceptées par différents partenaires. 
d'action précis. Dans deux des situations analysées (des organisations de création assez récente), les responsables de «la communication » affirmaient disposer d'un mandat large et en même temps se trouver dans la nécessité de créer une sphère d'action personnelle en interne et en externe pour mener à bien leur «mission». Dans le cas d'autres entreprises, le «DIRCOM» ou le responsable de la communication se situe à l'intérieur d'une équipe de cadres sous la responsabilité du sommet directorial.

\section{Les interlocuteurs et le contexte social}

Qui est le client ou l'utilisateur? La réponse formulée peut indiquer une source de légitimité et un, ou, de manière plus exacte, différents modes de légitimation. Un des acteurs se demande lors de la phase d'analyse des récits ce qui détermine l'organisation ; l'expression utilisée à plusieurs reprises consiste à connaître « ce qui tient l'organisation », « le ciment » de celle-ci :

Est-ce le commercial ? Parle-t-on des produits et des humains sans différenciation? Avant on demandait de défendre l'image, maintenant on nous demande de rétablir une certaine crédibilité et cela pose à la fois la question des choix stratégiques et de la manière d'aborder une négociation : si la confiance n'est plus là, il faut travailler à la rétablir et cela prend du temps, nous le savons, dans la mesure où l'on ne raconte pas tout et n'importe quoi. Il faut connaître ses interlocuteurs et être respecté d'eux : montrer le sérieux de ce que l'on fait et du temps passé avec eux» ${ }^{1}$.

Selon les personnes interrogées, différentes fonctions émergent dans ce contexte. Elles correspondent à des métiers précisés par une définition des postes ou le contrat d'engagement, mais ce n'est pas toujours le cas :

A certains moments, il faut composer avec la presse, avec les pouvoirs publics, les autres membres de l'équipe, en essayant de ménager les uns et les autres... A d'autres moments on reçoit une tâche relativement précise à effectuer... Mais nous devons

1 Cette réflexion a été validée en particulier dans l'échange entre trois participants à la première séance de travail. Elle a été confirmée par les entretiens postérieurs. 
tenir compte d'un ensemble de partenaires et de contraintes de notre action!

L'élargissement de la sphère des acteurs du contexte ou environnement sociétal pose d'autres défis ou enjeux. Trois responsables interrogés en entretien collectif, ont réagi de manière affirmative aux propos tenus par un directeur de communication en entreprise : la validité, la vérité des propos tenus par le communicateur en organisation est mise en cause par rapport aux propos tenus par les journalistes. A titre d'exemple ce fragment d'entretien :

Les services très professionnels des grandes sociétés voient leur action mise en doute : le doute porte sur la véracité des discours émanant des grands groupes...La construction d'images n'apparaît pas légitime, mais il n'y a pourtant pas d'image idéale, il y a l'essai d'expliquer ce que l'on fait, y compris les logiques parfois opposées mais qui ne vont pas vers la disparition de l'organisation...

Il ne s'agit pas d'un couple antinomique « amour-haine » entre communicateur d'organisation et journaliste (certains ont d'ailleurs assumé le passage d'un métier à l'autre). La légitimation par le corps social paraît toutefois plus facile pour le journaliste que pour le communicateur d'organisation accusé d'être « la voix de son maître » : si l'un dit vrai, l'autre ment, nécessairement...

Cette conception peut évoluer en fonction de la surface sociale dont dispose tel ou tel acteur par rapport à tel autre : le potentiel de confiance et d'estime dont jouissent les personnes. La construction d'un sens commun peut par ailleurs être expérimentée, comme l'indique la seconde partie de l'extrait d'entretien, dans un contexte de coopération conflictuelle. Par rapport aux différents cadres évoqués, citons la problématique de la transformation d'une entreprise publique dans une région en déclin industriel, la pertinence d'une organisation considérée comme « un gouffre sans fond» au départ par différents partenaires régionaux, la méfiance vis-à-vis de l'efficacité d'un service public, l'incidence du passage d'un service public à une entreprise autonome...

Selon les entretiens, certaines dimensions de la communication sont peu prises en compte : par exemple, la dimension intégrative est souvent négligée dans la perspective gestionnaire par rapport à la dimension instrumentale. L'élaboration d'un plan de communication 
est trop souvent considérée par le sommet gestionnaire, , comme une «prouesse technique » ou « la réalisation d'un produit sans commettre d'erreur », selon cinq responsables de communication interrogés: nous comprenons « erreur technique » ou « erreur de destinataire ».

La fonction de responsable de communication d'organisation renvoie à différentes capacités : la connaissance des sujets traités (la pertinence du discours), l'intégration des diverses composantes du sujet présenté (la fiabilité) et l'enracinement dans l'expérience du contexte (la proximité du «terrain»). Les différents responsables interrogés insistent sur la dimension importante des contacts interpersonnels et sur la fonction d'accompagnement de «processus de négociation» ou «d'évolution de l'organisation » ( par la mise en place d'actions de formation, réalisation d'outils d'information interne et externe, la gestion de campagnes ou de plans de communication...).

L'évocation de ces contextes particuliers peut dès lors nous amener à parler de métiers en évolution ou en construction: nous allons en préciser certains contours à partir d'une première élucidation des fonctions assumées et d'une revendication de statut professionnel.

\section{Une « chambre des métiers en construction sans négriers $\gg$ ?}

A partir des exemples étudiés, nous avons constaté le caractère polymorphe des fonctions exercées par les personnes interrogées: certain(e)s ont parlé de «bricolage», d'autres de «complexité des tâches ». Pour les premiers, il s'agit de l'évocation de tâches dictées par le sommet de l'organisation, en fonction d'un agenda bousculé par l'actualité des rapports sociaux (crise dans l'organisation, panne, accident...). Pour les autres, il s'agit du rapport à l'espace-temps planifié : le contexte de l'action organisée laisse une certaine autonomie aux chargés de communication dans la structuration de leurs tâches ${ }^{1}$.

1 Cette structuration nouvelle peut être liée à la croissance de l'organisation dans un nouveau contexte spatio-temporel ( dimension transnationale des activités, augmentation significative du nombre d'usagers) et à la diversification des tâches. 


\section{Du modèle technicien au modèle d'intermédiaire social ?}

A des métiers différents peuvent correspondre différents modes de légitimation ( le journalisme d'entreprise, la direction de communication, le marketing, la GRH....). Dès lors se pose la problématique de l'investissement de fonctions différentes par les mêmes personnes : la même personne est-elle susceptible d'accomplir plusieurs missions? Cette polyvalence est-elle liée à une organisation de création récente ou au contexte de crise dans lequel de nombreux professionnels sont amenés à oeuvrer? En fait l'identification à un métier peut rejoindre le modèle technicien, c'est-à-dire un rôle centré sur la « fiabilité du produit » et la sécurité du processus de production comme l'attestent certains entretiens.

D'autres éléments de discours ou de récits font émerger un modèle « inter-acteurs », celui d'un professionnel chargé d'une mission intégrative dans l'organisation et d'un processus de réorganisation des relations perturbées. Il a notamment été question dans les entretiens, du rôle du communicateur dans la préparation des négociations; d'autres ont abordé la mise au point d'actions de formation du personnel pour affronter de nouveaux défis : la fin de monopoles de service public, de nouveaux processus de fusion, l'exigence de développement de plus d'autonomie dans le financement de l'organisation.

On pourrait dès lors identifier la position professionnelle du communicateur dans une situation paradoxale: la dépendance serait plus importante dans les missions confiées en fonction de la proximité du sommet stratégique; l'autonomie serait davantage vécue dans la construction des dispositifs d'information et de mise en relation des différents partenaires. ${ }^{1}$ La fonction du communicateur ne peut être séparée de la compréhension des différents partenaires de l'environnement de l'organisation: travailler dans la communication implique dans ce contexte une différenciation et une intégration des rôles. L'obligation de rendre des comptes, la volonté de vérifier l'exactitude des propos tenus peuvent s'insérer dans une nouvelle sphère de construction des

1 C'est le cas de services publics pudiquement appelés «entreprises publiques autonomes »; c'est le cas d'organisations nouvelles montées avec des capitaux publics et invitées à construire une logique d'indépendance progressive ( atteindre le « seuil de rentabilité ») par rapport au pouvoir initial. 
relations sociales dans laquelle la construction du sens s'opère dans le jeu structuré des interactions ${ }^{1}$.

\section{Modèle statutaire ou modèle statufié ?}

Les acteurs interrogés ont souligné l'absence de statut officiel pour le communicateur d'entreprise, à l'opposé du « journaliste d'entreprise » qui s'inscrit dans un corps professionnel auquel correspond une charte précise. Celle-ci balise le contexte dans lequel est affirmée la relation à d'autres acteurs personnels ou institutionnels :

Là on a un statut reconnu et les membres acceptent une déontologie du métier qui rend crédible le travail pratiqué. Tous les communicateurs d'entreprise ne sont pas membres de l'Association Belge de la Presse d'Entreprise, mais c'est une association reconnue : on ne fait pas n'importe quoi et si on s'inscrit dans ce cadre, on accepte les mêmes règles du jeu. Cela permet de différencier un style de propagande d'un travail correct d'écriture et d'information diffusée vers le public...

A cet égard, on a pu faire observer, non sans pertinence, qu'une charte ne pouvait être considérée de manière équivalente à un statut clair, défini par un cadre légal ou en agrément entre acteurs : le discours de sens commun fera état du statut reconnu par une commission paritaire ou un organisme public. A un statut est souvent reliée une certification obligatoire pour occuper un poste précis de travail : la terminologie associée est celle de la « sanction officielle ». Par contre, une charte n'a de valeur qu'à l'intérieur d'un consensus entre partenaires. Elle définit toutefois de manière symbolique forte, un lieu de références éthiques et normatives dans lequel les activités sont reconnues, sans nier certains effets de notoriété: la référence à un jury d'éthique, l'affichage de la qualité de membre de telle association peut contribuer à légitimer l'action du communicateur, par exemple le journaliste d'entreprise :

1 Cette nouvelle sphère pourrait être caractérisée par le passage de la limitation des partenaires ("shareholders ») à l'élargissement de cette catégorie («stakeholders»). Les responsables interrogés évoquent nombre et les différentes « catégories de partenaires » à contacter et à «ne pas négliger »pour garantir la crédibilité de l'ensemble. 
Les entretiens analysés font apparaître la position intermédiaire du communicateur dans le corps social ( la notion du mandat donné par la direction, la liberté relative de parole et d'écriture). Nos interlocuteurs ont mis en évidence « la capacité versus l'incapacité » de négocier leur statut alors que d'autres cadres disposent de ce pouvoir : le cadre commercial ne s'occupe pas du domaine de l'ingénieur de production. Le chargé de communication ou le « dircom » ne disposerait pas de la même zone d'autonomie parce qu'il est amené à « rendre compte de l'organisation », «parler au nom de l'entreprise »,voire « faire la vérité sur l'organisation $»^{1}$.

La construction du modèle de statut dans une organisation n'apparaît pas pour autant différente selon la taille ou l'âge de l'entreprise : de chaque côté les professionnels réclament à la fois plus d'autonomie de fonction et plus de définition normative. Ce phénomène que nous pourrions relire comme exemple classique de double contrainte peut toutefois être élucidé dans une approche moins radicale par la prise en considération de différents indicateurs de contexte. Le professionnel réclame certes un cadre juridique et normatif clair pour éviter la sanction négative (peur d'outrepasser ses fonctions et ses droits). Il estime toutefois que la définition du poste devrait garantir une plus grande capacité d'action : la demande par certains de reconnaissance de titres « d'ingénieur en communication » (par rapport à des fonctions d'exécution)ou «d'ingénieur social» ( par rapport au travailleur social « de base») trouverait ici certains éléments d'explication².

Certes, la non-définition peut signifier la liberté entière de construire sa fonction; elle peut toutefois, et le contexte sociétal recèle de nombreux exemples, signifier l'arbitraire de la définition par d'autres et le déni de la fonction dans des contextes de crise : les personnes interrogées ont longuement évoqué le silence total des communicateurs officiels avant la disparition de la Sabena. Il a été fait mention de la classique référence au « déficit de communication » lorsque les tenants

1 Cette dernière expression mériterait à elle seule une discussion et une analyse des représentations de cette « vérité d'organisation » et de l'expérience du monde partagée, validée qui permet d'élaborer le consensus ou la dispute sur la « réalité de l'organisation communicante».

2 On lira avec intérêt à ce propos l'ouvrage déjà cité, Faut-il brûler la gestion de compétences, pp.228-249 
de divers pouvoirs n'arrivent plus à faire reconnaître le bien fondé de leurs décisions.

Les réflexions qui précèdent méritent d'être replacées dans un nouveau contexte : l'évolution des relations de travail se déroule dans des mutations du rapport à la norme au sein du contexte de travail. Christian Maroy met en évidence le paradoxe suivant : nous assistons de manière simultanée à un renforcement des procédures de contrôle par rapport à l'écart de la norme (notamment dans les dispositifs de certification et de «qualité totale ») et à un large appel à l'autonomie des différents acteurs ${ }^{1}$.

Les professionnels de la communication apparaissent dans cette évolution sociétale, non comme une catégorie séparée d'autres corps professionnels, mais comme des éléments particuliers d'un vaste ensemble dans lequel n'émerge plus de modèle univoque de référence dans un contexte donné ${ }^{2}$. La position professionnelle du communicateur apparaît de manière plus précise dans la sphère de contacts entre des acteurs qui n'occupent pas la même place et ne disposent pas de la même capacité d'action dans le jeu structuré de l'action organisée. Un fragment d'entretien peut nous éclairer à ce propos :

Il ne faut pas seulement considérer le métier mais aussi la segmentation des fonctions, en considérant qu'il y a des différences de positionnement social : de qui dépend-on ? Qui donne la mission, le mandat? On se trouve à ce moment en train de bricoler un rôle tout en espérant que cela ne va pas se retourner contre nous $! . .{ }^{3}$

1 C.MAROY, «Rapport à la norme et transformation des modes d'organisation de la production et du travail dans l'entreprise », in J.DE MUNCK, M.VERHOEVEN et al., "Les mutations du rapport à la norme. Un changement dans la modernité »,Paris, Bruxelles, De Boeck Université, 1997,pp.107-120. L'auteur analyse des enquêtes réalisées dans les entreprises informatisées ou en pleine transformations des processus de production.

2 V. dans l'ouvrage déjà cité, la préface de André BERTEN, en particulier quand celui-ci énonce : "Du point de vue de la coordination de l'action, il faut dès lors abandonner la représentation d'un monde qui fournirait le cadre le plus général de ce qu'il faut faire ou ne pas faire et, en conséquence, la question de la coordination est renvoyée aux acteurs eux-mêmes. Or ceux-ci se découvrent de plus en plus comme des coopérants pragmatiques, partageant de façon stratégique, ponctuelle ou durable, un ou plusieurs projets partiellement communs »(p.7)

3 Cette question du mandat a été abordée par la plupart des acteurs interrogés. Tous 
Cette situation n'est pas le pur résultat de la détermination normative d'un «poste de travail» mais l'effet des tensions relatives aux tâches exercées. La personne chargée de fonctions de communication est confrontée à des exigences de fidélité au mandat confié par le sommet de l'organisation et de crédibilité face aux différents acteurs mis en scène dans des contextes délicats (tels le processus de négociations difficiles ou l'annonce de changements brusques dans un contexte local ou régional).

La double position du communicateur d'entreprise ne renvoie pas à une duplicité des chargés de communication mais bien à la position particulière de l'acteur social étudié et aux ressources disponibles déjà présentées: la maîtrise de moyens d'information et la capacité de les utiliser par rapport à des situations sociales variables (le modèle technicien) ; la capacité de mettre en commun différents partenaires dans un système de coopération conflictuelle (le modèle inter-acteur) ${ }^{1}$.

Dans l'état actuel de la recherche, nous estimons qu'une combinaison de différents facteurs humains contribue à la force du lien construit avec les interlocuteurs et donc à la pertinence du rapport explicite : qu'il s'agisse de présenter l'état de négociations sociales dans une organisation en pleine restructuration, ou de défendre la validité d'une politique de dépenses publiques dans un ensemble situé dans une région en déclin économique, les qualificatifs adoptés sont ceux de « fiable», « juste », « adéquat».

Par ailleurs, dans cette recherche, il est essentiel d'aborder un autre champ de référence que celui de l'organisation proprement dite : dans le contexte sociétal, plusieurs responsables font référence dans des discours ou des écrits, à des traits conceptuels redondants tels les qualificatifs de « novateurs », « porteurs de progrès », « porteurs d'avenir ...». La communication conférerait une valeur ajoutée à l'organisation, elle permettrait de valoriser la place de l'organisation dans un contexte sociétal précis. Que le professionnel de la communication soit reconnu dans des capacités purement techniques, dans un rôle d'intermédiaire

ont mis en évidence également le caractère délicat de l'intermédiaire pris entre différentes logiques de jeu et positions d'acteurs institutionnels.

1 L'attestent différents témoignages recueillis : la qualification du caractère «flou » du travail à effectuer dans des circonstances difficiles, et l'affirmation de «l'indétermination de l'avenir des organisations étudiées ». 
entre différents acteurs sociaux ou dans cette double qualité, nous ne pouvons éluder la question de la valeur, du coût de l'expertise et validée par les acteurs d'un contexte plus vaste (ce que l'on nomme de manière habituelle « la société civile »...)

\section{Pouvoir communiquer : le prix et le cout de l'expertise}

\section{Un pouvoir interpersonnel ?}

Le pouvoir acquis paraît, selon nos différents interlocuteurs, lié largement à la conjonction des capacités techniques et de la surface relationnelle développée. En termes et en champs de recherche différents nous renverrons au concept de capital social chez Bourdieu et à la notion de maîtrise des zones d'incertitude chez Crozier et Friedberg: nous faisons référence à la capacité d'entrer en réseau avec d'autres et de développer des coalitions particulières d'acteurs pour faire reconnaître la pertinence de telle ou telle démarche ${ }^{1}$. Il peut s'agir de telle action proposée dans un rapport divergent des projets du sommet de l'organisation ou d'un projet élaboré au sein de rapports conflictuels entre des acteurs ( cadre de négociations, campagne de communication dans un contexte de restructuration etc...).

La problématique des procédures de communication interne et externe auxquelles faisait référence la demande de statut clair est à étudier dans ce cadre plus large : les procédures codifiées (directives, cadre de loi ou coutume établie...) n'ont de sens que si elles sont connues et reconnues dans un contexte social délimité.

L'atout particulier des professionnels dans certains contextes est apparu lié à la capacité de mobiliser des ressources matérielles et de coaliser des acteurs par rapport à des intérêts collectifs précis : à qui

1 On lira à ce propos avec intérêt l'ouvrage collectif sous la direction de A. BEVORT et M. LALLEMENT, Le capital social, Performance, équité et réciprocité, Paris, Editions de la Découverte, MAUSS, 2006. La notion du capital social y est analysée à nouveaux frais sous divers aspects, notamment au plan de la légitimité construite par la qualité des relations sociales construites chez divers acteurs sociaux. Cette réflexion s'inscrit dans le propos général des membres du mouvement antiutilitariste dans les sciences sociales (M.A.U.S.S.). Voir également M. CASTELLS, "La société en réseaux », Paris, Fayard, 1998 
s'adresse-t-on ? De quelle manière ? Avec qui ? Pour aboutir à quoi ?La légitimation des acteurs se construit dans un jeu croisé de relations et d'actions conjointes; elle renvoie dès lors à un autre élément : la régulation des différents flux d'information et l'accompagnement des processus de réflexion, de discussion et de décision. C'est en ce sens que l'on peut parler du rôle des experts et des intermédiaires que sont les communicateurs des organisations à l'interface du secteur public et du secteur privé.

\section{Un pouvoir d'expertise?}

Nos différents interlocuteurs ont mis en évidence les enjeux du travail de «communicateur» dans des contextes de choix difficiles à opérer, de politiques à construire, d'intermédiaires entre acteurs passionnés par des éléments divergents : rénovation industrielle versus extension de la sphère de production ; modernisation d'un site de production versus diminution de l'emploi ou absence de croissance de celuici ; construction d'un ensemble culturel de prestige versus dépenses importantes dans une région touchée par la récession économique. Le travail s'opère dans une société complexe, en mal de redéfinition et en l'absence de critère absolu. En ce sens le rôle «d'accompagnateur » des processus de discussion et négociation mérite d'être quelque peu analysée dans les limites de l'espace disponible ici.

Les professionnels de la communication, selon les indices relevés, sont appelés à déchiffrer les éléments de contexte des pratiques volontaires de communication ( les politiques de communication) et involontaires ( les phénomènes de communication, l'ensemble des actes de communication formelle et informelle) qui traversent et structurent l'organisation. C'est à ce prix qu'ils et elles peuvent assumer leurs rôles dans un contexte sociétal traversé par des tendances diverses que l'on qualifiera de « replis identitaires » ou de « fuite en avant », s'il s'agit d'un côté de référence à des rôles déjà recensés ou, d'un autre côté, de fonctions relevant de l'invention pure : dans le jeu des diverses interventions reconnues par les membres de la société civile ou frappées de soupçon, le rôle est à la fois défini dans un contexte précis et dans la capacité de construire du sens pour le corps social concerné...

La construction des métiers énoncés ( DIRCOM, responsable de communication externe ou interne) ne renvoie dès lors pas simplement 
à un arbitraire du mandat ou de la délégation, mais à la capacité de construire un dispositif de rencontres dans le système de coopération conflictuelle : d'où l'insistance sur le «travail à fournir », « le sérieux du métier » ou « l'accompagnement » des négociations. La dépendance d'une direction générale, qu'il s'agisse d'une grande entreprise mixte, ou de pouvoirs publics à l'échelon régional, inclut la capacité de mettre en évidence des enjeux, de préciser les éléments principaux de convergences et de divergences.

Nous avons pu faire émerger une double caractéristique chez les communicateurs professionnels :d'une part la capacité de construire des réseaux de relations générateurs de confiance mutuelle; d'autre part le pouvoir d'expertise dans un contexte particulier (rendre compte de la réalité vécue et du conflit des interprétations). Nous proposons dès lors d'énoncer certains enjeux de recherche à poursuivre pour cerner avec finesse et rigueur l'évolution de métiers cités ou émergents.

\section{Quelques questions et enjeux}

Les réflexions émises lors des entretiens et de l'analyse ex post ont fait référence aux mutations dans l'appartenance de nouveaux engagés et dans la construction du métier : il a été fait mention de la logique de contrat plutôt que d'adhésion à l'organisation conçue comme un prolongement de l'identité personnelle. Nous ne rappellerons pas ici l'ensemble des travaux de Sainsaulieu et la différenciation des modes d'appartenance à une organisation dans le contexte occidental postindustriel (la prégnance de la logique du contrat et de l'adhésion limitée) ${ }^{1}$. Nous allons toutefois reprendre certains éléments en termes de capacités ou de compétences mises en œuvre dans des métiers de la communication, sans prétention d'exhaustivité.

Nous renvoyons tout d'abord à la problématique de l'accompagnement des négociations, des prises de décision ou de la structuration des relations entre différents acteurs, les «parties prenantes » de l'action. Les entretiens ont fait émerger différentes capacités ou compétences d'action : la capacité d'opérer des contacts entre divers acteurs, celle

1 R.SAINSAULIEU, « Sociologie de l'entreprise », Paris, Seuil, 1998 
de livrer des informations précises sur le contexte, de permettre les flux d'information entre protagonistes. En mineure est apparue une capacité non imaginée au départ : celle d'intervenir dans un processus de formation et d'éducation permanente. La mission d'accompagnement évoquée par différents partenaires inclut la vérification des expériences vécues dans un contexte de mutations, par différents acteurs de l'organisation (vision intégrative au-delà de la segmentation des différentes fonctions) $)^{1}$.

Ces constats nous amènent à valider une proposition de logique d'intégration des fonctions plutôt que d'une logique de segmentation : logique de profession plutôt que de métiers pleinement différenciés (même si la personne est amenée à changer de fonctions au cours de son existence professionnelle).

La capacité d'intervention peut également englober la facilitation d'expression des uns et des autres dans un cadre conflictuel plus important: le processus de structuration des relations dans un contexte de divergences profondes ou de dissentiment et de préparation des négociations implique tout un travail de l'organisation sur elle-même. C'est dans ce contexte que différents auteurs parlent de «l'entreprise apprenante » et du rôle que peuvent y jouer les responsables de communication $^{2}$.

Pour mettre en œuvre ces processus, les acteurs sont censés mobiliser différentes compétences non seulement techniques mais également organisationnelles, des compétences d'expertise et de gestion des situations relationnelles, mais également de prospective et d'anticipation des conflits potentiels. Cette capacité prévisionnelle est-elle envisageable dans un contexte sociétal évolutif ou faut-il considérer que la communication en temps ordinaire inclut la communication de crise comme dimension constitutive de la fonction ? Ne peut-on, à la suite de nos différents interlocuteurs, considérer le dispositif de légitimation

1 Cette représentation des métiers et de la profession de communicateur a été abordée comme mode de sortie des impasses vécues par les responsables de communication dans trois entreprises publiques.

2 Voir à ce propos notamment les travaux de G.MINGUET et de G.HERREROS, en particulier G.HERREROS, L'intervention du sociologue revisitée, Education Permanente, Formation des adultes, : de l'utopie à la crise , 129, 129-148,1996. voir également les réflexions de P.VENDRAMIN, "Le travail au singulier", Louvain la Neuve, Academia Bruylant, 2004, en particulier pp.222-228. 
de la profession dans la mise en oeuvre d'un réseau de coopération conflictuelle ?Nous avons tenté de synthétiser la mise en évidence de ce processus. Nous estimons qu'une étude ultérieure pourrait se concentrer sur une logique sectorielle pour cerner certains contours spécifiques à des contextes particuliers d'organisation ( les services marchands ou non marchands, la production et la gestion culturelle...)

Citons enfin les conflits de représentations et la gestion de ces conflits ainsi que les modes de production sociale et de régulation des actions de communication : ces capacités font référence au double caractère de la loi, « de lege acta et ferenda », c'est-à-dire aux dimensions instituée et instituante de toute activité sociale. Le chantier reste ouvert pour affiner l'approche de ce groupe social intermédiaire des professionnels en communication d'organisation dans un contexte sociétal en pleine mutation : nous pensons en particulier au caractère transnational des regroupements d'entreprises ou de services et à l'absence de capacité régulatrice d'organismes supranationaux. 